Colloids and Surfaces

A: Physicochemical and Engineering Aspects 128 (1997) 255-264

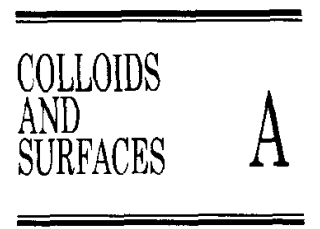

\title{
Flexible membranes with anchored polymers
}

\author{
Reinhard Lipowsky \\ MPI für Kolloid- und Grenzflächenforschung, Kantstr. 55, 14513 Teltow, Germany \\ Received 12 August 1996
}

\begin{abstract}
Compound systems are considered which consist of flexible membranes and polymers. The polymer chains are attached to the membrane surfaces via anchor segments. The non-anchored segments of the polymers, on the other hand, may be repelled from or attracted toward the membranes which leads to desorbed mushrooms or adsorbed pancakes, respectively. These anchored polymers change the local curvature of the membranes. In addition, they can act as adhesive stickers between membranes, a mechanism which underlies the adhesion of biomembranes. (C) 1997 Elsevier Science B.V.
\end{abstract}

Keywords: Membrane curvature; Polymer mushrooms and brushes; Adsorption transition; Adhesion via stickers

\section{Introduction}

During the last two decades, much effort has been devoted to the study of flexible membranes composed of amphiphilic molecules. As a result of these efforts, we now understand various aspects of their self-organization and of their cooperative behavior at the supramolecular level [1]. At the same time, the physics of polymers has also been further developed [2-4]. These two research fields have remained rather separate, however, even though the behavior of membranes and polymers exhibits many similarities. For example, both polymers and membranes are often highly flexible and exhibit strong fluctuation effects.

A new level of self-organization is found in systems which are composed of both membranes and polymers. In this paper, I review our recent work on such systems [5-8]. The polymers are attached to the membranes by anchor segments which insert into the membranes. The same mechanism is effective for biopolymers and biomembranes $[9,10]$. Two general aspects related to the flexibility of these objects will be emphasized: (i) anchored polymers induce a spontaneous curvature of the membranes [5-7], and (ii) the adhesion of membranes mediated by sticky polymers exhibits a threshold behavior arising from the membrane fluctuations [8].

This paper is organized as follows. In this introductory section, some basic features of membrane/polymer systems are described. The following Sections 2 and 3 contain short summaries of our work on polymer-induced curvature and polymer-induced adhesion, respectively.

\subsection{Biomembranes as lipid/polymer compounds}

Biomembranes are very thin molecular sheets which consist of a lipid bilayer and a large number of amphiphilic macromolecules. The latter molecules, which are anchored in the membranes, are usually connected to a network of relatively stiff, rod-like filaments which belong to the so-called cytoskeleton within the cell. Likewise, the outer plasma membrane is often covered by a "coat" of 
polysaccharides, i.e. of branched polymers which are also anchored within the membranes. These systems can be modelled, to a certain extent, by the decoration of lipid bilayers with polymers which are anchored in the bilayers.

Cell membranes and other biomembranes are typically in a fluid state. Therefore, the model membranes considered here are also taken to be fluid (with the exception of the last Section 3.3). Fluid membranes are very flexible and can easily change their shape. Thus, in contrast to a solid substrate or any other interface, such a membrane can easily respond and adapt to the interactions with the polymers.

For fluid membranes, both the lipid molecules and the anchored polymers are usually mobile and can move along the membranes. In some cases, the anchored polymers may be immobilized when they are bound to another surface or to an external polymer network (such as the cytoskeleton). The case of immobile polymers will be briefly discussed in Section 3.3.

\subsection{Polymers with anchors}

The simplest polymer architecture is provided by linear chains. Since the solvent is taken to be aqueous, all monomers apart from the anchor segments must be water-soluble or polar $(\mathrm{P})$. The anchor (A) segments, on the other hand, have to penetrate into the hydrophobic interior of the lipid bilayer and, thus, must consist of a hydrophobic or non-polar block or segment, the size of which is adapted to the thickness of the lipid bilayer. Now, one may have (i) an AP...P chain with one anchor at its end, or (ii) an AP...PA chain with one anchor at both ends, or (iii) a P...PAP...P chain with an interior anchor segment, etc.

In Section 3, I will also consider monomer sequences of the form AP...PS where S is a sticky segment. Such a segment may adhere directly to another membrane, may form a direct bond with another S' segment, or may be connected to another S' segment via a linker molecule.

One type of anchor which is well adapted to the lipid bilayer is provided by a lipid molecule; the polymer is then covalently bound to the head group of such a molecule (Fig. 1). Such lipid anchors have been realized artificially, e.g. for

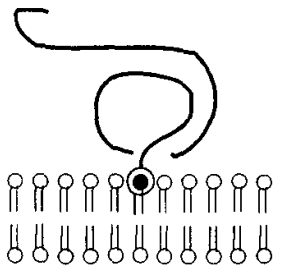

(a)

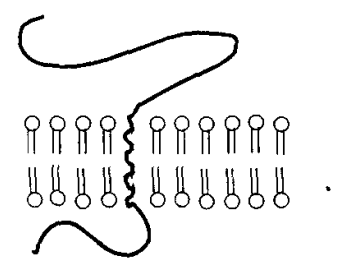

(c)

Fig. 1. Different ways to attach polymer chains to bilayer membranes: (a) lipid anchor; (b) hydrophobic side chain; (c) membrane-spanning polymer segment; and (d) anchored and adsorbed chain.

poly(oxyethylene) also known as polyethylene glycol (PEG) [11-13], These anchors are also used in biological systems, for example in order to attach cell adhesion molecules (such as N-CAM 120) to cell membranes $[9,10]$. For such anchors, the whole polymer chain is located on one side of the membrane.

Another type of anchor which has been used in model systems is provided by hydrophobic sidegroups of the polymer (Fig. 1). One example is provided by amphiphilic copolymers consisting of segments of poly( $N$-isopropylacrylamides) and of monomers with hydrophobic sidegroups for which many different sequences have been synthesized $[14,15]$. Such a polymer presumably inserts many anchors into the bilayer and, thus, forms a flexible comb which is again located on one side of the membrane. Membrane proteins, i.e. amino acid chains, on the other hand, are often anchored by a membrane spanning segment which contains amino acids with hydrophobic residues. In the latter case, the polymer chain stands out from both sides of the lipid bilayer (Fig. 1).

\subsection{Polymer-membrane interactions}

Lipid bilayers have a thickness of about 4-5 nm and often have lateral dimensions of many $\mu \mathrm{m}$. 
For synthetic polymer chains, the number $N$ of statistical segments usually varies in the range $10^{2} \lesssim N \lesssim 10^{5}$. If the chain is highly flexible, this number is equal to the number of monomers and the persistence length $a_{\mathrm{po}}$ is comparable to the size of the monomer. In good and $\theta$ solvents, these chains form random coils with a linear size

$R_{\mathrm{po}} \simeq a_{\mathrm{po}} N^{v}$ with $v \simeq 3 / 5$ and $v=1 / 2$, respectively

See, for example, Ref. [2]. For $a_{\mathrm{po}} \simeq 0.3 \mathrm{~nm}, 10^{2} \lesssim$ $N \lesssim 10^{5}$ leads to $5 \mathrm{~nm} \lesssim R_{\text {po }} \lesssim 300 \mathrm{~nm}$ for good solvent conditions. Thus, in a typical situation, the dissolved polymer has a linear size which is larger than the thickness of the lipid membrane but small compared to its lateral dimensions. When anchored to the membrane, the polymer will interact with a membrane segment, the size of which is comparable to $R_{\text {po }}$

In general, the interactions between the polymer and the membrane will have many contributions arising from molecular forces which can be repulsive or attractive. If the non-anchored segments of the polymer are effectively repelled from or attracted to the membrane surface, the anchored polymer is in a desorbed or in an adsorbed state, respectively. In the desorbed state, a polymer which is attached to the membrane by a single anchor has the form of a mushroom, the size of which is comparable to the dissolved polymer. Weak adsorption leads to squashed mushrooms; strong adsorption to polymer pancakes which are tightly bound onto the membrane surface (a similar terminology has been used for polymers grafted onto solid surfaces).

\subsection{Separation of time scales}

The membrane/polymer system involves several time scales which will now be estimated for the mushroom case. One time scale is related to the possibility that the anchor may be uprooted from the membrane [16]; in this case, the polymer is no longer attached to the membrane and escapes into the surrounding solution. Indeed, the mushroom state is governed by a finite anchoring free energy $\Delta F_{\text {an }}$ and, thus, by a corresponding residence time $t_{\text {res }} \sim \exp \left(\Delta F_{\text {an }} / T\right)$ at temperature $T$ (here and below, temperature is measured in energy units, i.e. $T$ is a short-hand notation for the Boltzmann constant $k_{\mathrm{B}}$ times temperature).

The anchoring free energy $\Delta F_{\text {an }}$ of the polymer consists (i) of the energy gained by inserting the anchor into the bilayer membrane and (ii) of the entropy lost by constraining the chain to one side of the membrane surface. For polymers which are anchored via phospholipid anchors, the anchoring free energy is dominated by the anchoring energy which is large compared to $T$ : for dipalmitoyl phosphatidyl choline (DPPC) anchors, for example, one has an anchoring energy of $\simeq-27 T$ at room temperature [5]. In such a situation, the residence time $t_{\text {res }}$ of the anchored polymer may be estimated by the residence time of the phospholipid molecules. The latter time can be determined experimentally and is typically found to be of the order of several hours (this time will increase if the interaction between the membrane surface and the non-anchored polymer segments becomes more attractive).

If the polymer and the membrane segment have been bound together, the approach towards their equilibrium states is characterized by certain relaxation times $t_{\mathrm{pol}}$ and $t_{\mathrm{mem}}$, respectively.

In solution, the relaxation of a single polymer coil of linear size $R_{\mathrm{po}}$ is governed by hydrodynamic interactions which leads to the so-called Zimm dynamics and to the relaxation time $t_{\mathrm{pol}} \simeq \eta R_{\mathrm{po}}^{3} / T$ (see, e.g., Ref. [3]) where $\eta$ is the dynamic viscosity of the solvent. For water, one has $\eta \simeq 10^{-3} \mathrm{~J} \mathrm{~s} \mathrm{~m}^{-3}$. At room temperature, this leads to $t_{\mathrm{pol}} \simeq\left(R_{\mathrm{po}} / \mu \mathrm{m}\right)^{3} \times 10^{-1} \mathrm{~s}$. This estimate also holds for an anchored polymer in the mushroom state as follows from dimensional analysis (the prefactor will be different and may be calculated using the appropriate Oseen tensor). It implies that the relaxation time $t_{\mathrm{pol}}$ lies in the range $10^{-8} \mathrm{~s} \lesssim t_{\text {pol }} \lesssim 3 \times 10^{-3} \mathrm{~s}$ for polymers with $5 \mathrm{~nm} \lesssim R_{\mathrm{po}} \lesssim 300 \mathrm{~nm}$

The dynamic relaxation of the curvature of a bilayer membrane, on the other hand, involves several dissipative processes. On sufficiently large length scales $L_{\|}$, the energy is primarily dissipated in the surrounding liquid which is governed by the relaxation time $t_{\text {mem }} \simeq \eta L_{\|}^{3} / \kappa$, where $\kappa$ is the bend- 
ing rigidity of the membrane [17]. For lipid bilayers with $\kappa \simeq 10^{-19} \mathrm{~J}$, this applies to length scales $L_{\|}>1 \mu \mathrm{m}$. For smaller length scales, the slowest process is given by the relaxation of the density difference between the two monolayers and is thus governed by intra-monolayer friction $[18,19]$, In this regime, the relaxation time $t_{\text {mem }}$ of a bending deformation with linear size $L_{\|}$can be estimated as $\left(L_{\|} / \mu \mathrm{m}\right)^{2} \times 10^{-2} \mathrm{~s}$. Using these estimates, the relaxation time $t_{\mathrm{mem}}$ for a curved membrane segment with curvature radius $L_{\|} \simeq R_{\text {po }}$ and $5 \mathrm{~nm} \lesssim R_{\text {po }} \lesssim 300 \mathrm{~nm}$ will be in the range $10^{-7} \mathrm{~s} \lesssim t_{\text {rel }} \lesssim 10^{-3} \mathrm{~s}$.

Therefore, for phospholipid anchors, the residence time $t_{\text {res }}$ is always large compared to the relaxation times $t_{\mathrm{pol}}$ and $t_{\mathrm{mem}}$ (with $t_{\mathrm{pol}}>t_{\mathrm{mem}}$ for long chains) and the mushroom state has sufficient time to attain its equilibrium state. Such a separation of time scales should apply to all states of the polymer/membrane system considered in the following as long as the polymer coverage is not too large.

\section{Polymer-induced curvature of membranes}

In general, the polymers will be attached to both sides, i.e. to both monolayers of the lipid bilayer. In practice, the anchor concentration in each monolayer can be varied by changing the polymer concentration in the adjacent solution and by changing the time over which the monolayer is exposed to this solution. The polymer coverage arising from this exposure of each monolayer depends both on the number of attached anchors and on the chain length of the anchored polymers.

If both monolayers exhibit a different polymer coverage, the decorated bilayer is asymmetric and will then exhibit a polymer-induced spontaneous curvature. As discussed in the following, this curvature $M_{\mathrm{pi}}$ depends (i) on the size of the polymer coil which varies, for example, with the solvent conditions, (ii) on the polymer coverage, and (iii) on the interactions between the membrane surface and the non-anchored polymer segments.

The concept of spontaneous curvature was introduced some time ago for monolayers [20] and for bilayers [21]. It is both appealing from an intuitive point of view and also represents a fundamental aspect of thermodynamics since it is conjugate to the total mean curvature, one of the basic measures of integral geometry [22,23]. However, in most cases, the magnitude of the spontaneous curvature is rather difficult to estimate since it depends on many molecular parameters. In contrast, the spontaneous curvature induced by anchored polymers depends only on a few parameters which are experimentally accessible, as described in the following subsections.

\subsection{Repulsive polymer-membrane interactions}

\subsubsection{Dilute regime and mushroom-induced curvature}

First, consider a single mushroom of an AP...P chain, i.e. of a linear chain for which the anchor segment A is located at one of its ends and for which the non-anchored polymer segments experience effectively repulsive interactions with the membrane surface.

Such an anchored polymer tends to exert entropic or fluctuation forces onto the bilayer membrane which bend the membrane away from the polymer (Fig. 2). The resulting polymerinduced mean curvature is given by [5]

$M_{\mathrm{pi}} \sim+T / \kappa R_{\mathrm{po}} \sim+T / \kappa a_{\mathrm{po}} N^{v}$

A linear chain with a P...PAP...P sequence, i.e. with an interior anchor segment $\mathrm{A}$, gives rise to two mushrooms which stand up on both sides of the bilayer. If the two exterior chain segments consist of $N_{\mathrm{a}}$ and $N_{\mathrm{b}}$ statistical segments, these mushrooms have linear size $R_{\mathrm{a}} \sim N_{\mathrm{a}}^{v}$ and $R_{\mathrm{b}} \sim N_{\mathrm{b}}^{v}$, respectively. If the two sizes $R_{\mathrm{a}}$ and $R_{\mathrm{b}}$ are comparable, this leads to the induced curvature $M_{\mathrm{pi}} \sim T\left(R_{\mathrm{a}}-R_{\mathrm{b}}\right) / \kappa R_{\mathrm{b}}^{2}$, where $R_{\mathrm{a}} \geq R_{\mathrm{b}}$ has been

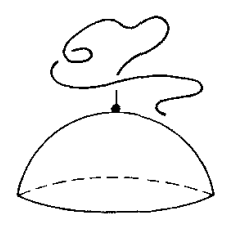

SPHERE

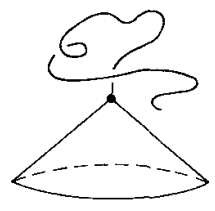

CONE

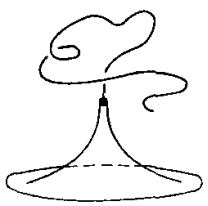

CATENOID
Fig. 2. Polymer mushroom anchored at membrane segments of different shapes. 
assumed. If the two mushrooms are very different in size, the membrane will attain a more complex shape since the larger mushroom interacts with a larger area of the membrane surface.

In the above estimates, the effect of the anchored polymer on the bending rigidity $\kappa$ and on the Gaussian bending modulus [21] $\kappa_{\mathrm{G}}$ has been ignored. A small curvature expansion up to second order in the curvatures leads to the effective bending rigidity $\kappa_{\mathrm{eff}}=\kappa+c_{1} T$ and to the effective Gaussian bending modulus $\kappa_{\mathrm{G} \text {,eff }}=\kappa_{\mathrm{G}}+c_{2} T$, where the dimensionless coefficient $c_{1}$ is positive whereas $c_{2}$ is negative [6]. For ideal polymers, one finds $c_{1} \simeq+0.21$ and $c_{2} \simeq-0.17$. Thus, for lipid bilayers with $\kappa \simeq 10^{-19} \mathrm{~J}$, the mushroom-induced change of the bending rigidity is rather small.

\subsubsection{Overlap coverage and semi-dilute brush regime}

For each monolayer, the polymer coverage can be defined by $\Gamma \equiv N / \xi_{\text {an }}^{2}$, where $\xi_{\text {an }}$ describes the mean distance between the anchors or the mean grafting distance. When $\xi_{\text {an }}$ becomes comparable to the size $R_{\mathrm{po}}$ of the mushrooms, the polymers start to overlap and to squeeze one another. They then attain conformations which are stretched away from the surface and resemble brush states (Fig. 3).

The overlap coverage corresponding to $\xi_{\mathrm{an}}=R_{\mathrm{po}}$ is given by $\Gamma_{\mathrm{ov}}=N^{1-2 v} / a_{\mathrm{po}}^{2}$. In the dense limit, one obtains the limiting value $\Gamma_{\mathrm{de}}=N / a_{\mathrm{po}}^{2}$ for the coverage (which will not be reached in practice since the entropy loss arising from the steric hindrance by the neighboring polymers within the brush increases with the coverage and eventually overcomes the anchoring energy). It is then convenient to introduce the reduced coverage $\bar{\Gamma}$ defined by $\bar{\Gamma} \equiv I / \Gamma_{\mathrm{de}}=\left(a_{\mathrm{po}} / \xi_{\mathrm{an}}\right)^{2}$ which is essentially the area fraction of the monolayer surface covered by anchors. The corresponding overlap coverage is $\bar{\Gamma}_{\text {ov }}=1 / N^{2 v}$

If the brush is again anchored on one side of the membrane, the membrane bends away from the brush and exhibits a polymer-induced curvature $M_{\mathrm{pi}}$ which increases monotonically with the coverage $\bar{\Gamma}$. For good solvent conditions, scaling arguments based on the blob picture [24-26] lead to $M_{\mathrm{pi}} \sim N^{2} \bar{\Gamma}^{13 / 6}$ and $M_{\mathrm{pi}} \sim N^{1 / 7} \bar{\Gamma}^{13 / 21}$ for small and large $\bar{\Gamma}$, respectively [6], where the Flory value $v=3 / 5$ has been used.

As in the case of mushrooms, the polymer brush acts to increase the bending rigidity $\kappa$, but to decrease the Gaussian bending modulus $\kappa_{\mathrm{G}}$ Using the aforementioned scaling picture, one finds [6] $\kappa_{\text {eff }}=\kappa+\left[(v+2) / 12 v^{2}\right] N^{3} \bar{\Gamma}^{3 / 2 v} T$ and $\kappa_{\mathrm{G}, \text { eff }}=$ $\kappa_{\mathrm{G}}-(1 / 6 v) N^{3} \bar{\Gamma}^{3 / 2 v} T$. The same signs for the excess rigidities are found if one uses mean-field theories for the brush as developed, for example, in Ref. [27].

These results can be extended to bilayers which are decorated in such a way that both monolayers are covered by a brush. Within a small curvature expansion, the contributions of these two brushes to the free energy are additive. Thus, for the

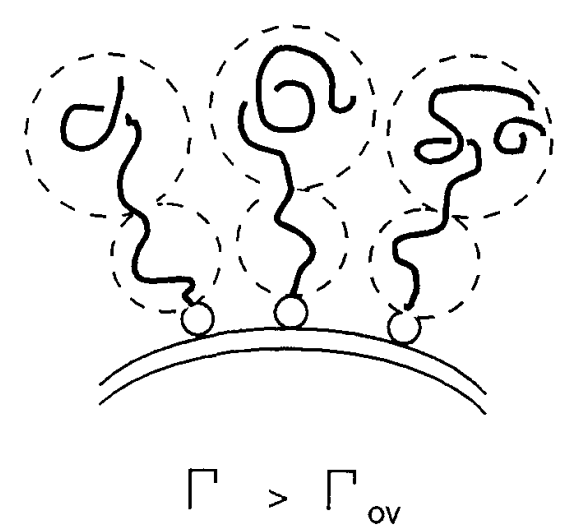

Fig. 3. Curvature induced by anchored polymer brushes: (left) brush at the overlap concentration $\Gamma \simeq \Gamma_{\text {ov }}$; (right) brush in the semidilute regime with $\Gamma>\Gamma_{\mathrm{ov}}$ 
symmetric case, in which the brushes on both sides have the same density, the polymer-induced curvature vanishes and the polymer-induced contribution to the bending rigidities is twice the contribution induced by a single brush. The symmetric situation has also been studied in Ref. [28] where similar results were found from a somewhat different scaling approach.

\subsection{Attractive polymer-membrane interactions}

\subsubsection{Pancake-induced curvature}

Now, let us consider again a linear AP...P chain with anchor segment $\mathrm{A}$ inserted into the bilayer membrane. However, the non-anchored segments $\mathrm{P}$ now experience a more complex interaction with the membrane surface: apart from a hard wall interaction, which reflects the fact that the $\mathrm{P}$ segments cannot penetrate the bilayer, these segments also experience attractive interactions with the membrane surface. If the strength of the attractive potential well exceeds a certain threshold, the polymer will be adsorbed onto the membrane.

In the strongly adsorbed state, the anchored polymer forms a pancake as mentioned. It turns out that the membrane now bends towards the polymer in order to maximize the number of contact points with the pancake. Therefore, such a pancake induces a spontaneous curvature which has the opposite sign compared to the mushroom case (Fig. 4). Using scaling arguments and explicit calculations for contact potentials, the pancakeinduced curvature is found to scale as [7]

$M_{\mathrm{pi}} \sim-T / \kappa L_{\perp}$

where the relevant length scale is now given by the pancake thickness $L_{\perp}$ which replaces the mushroom
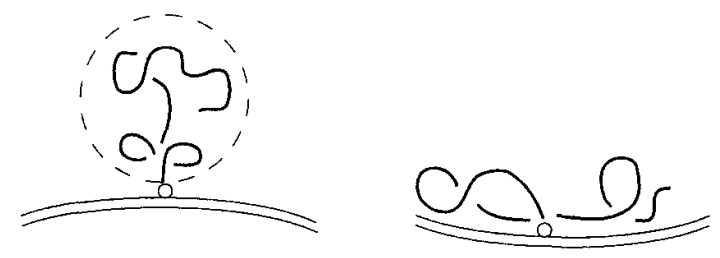

Fig. 4. An anchored polymer which undergoes an adsorptiondesorption transition induces a downward curvature $M_{\mathrm{pi}}>0$ for desorption (left), and an upward curvature $\boldsymbol{M}_{\mathrm{pi}}<0$ for adsorption (right). size $R_{\text {po }}$ in Eq. (2). In Ref. [29], the same parameter dependence of $M_{\mathrm{pi}}$ has been proposed, but with the opposite sign. The sign as given in Eq. (3) is consistent with the results of mean-field theory when applied to many-chain adsorption [30].

\subsubsection{Adsorption-desorption transition}

In general, the anchored polymer may undergo an adsorption-desorption transition as one varies the temperature or the solvent conditions (such as, for example, the $\mathrm{pH}$ ). In such a situation, the polymer transforms from a pancake to a mushroom and the curvature of the adjacent membrane segment changes its sign (Fig. 4). Therefore, anchored polymers close to such a transition provide a kind of "curvature switch".

In order to discuss the transition region in more detail, let us be more precise and let us consider an attractive interaction potential between the membrane surface and the non-anchored segments of the polymer which is short-ranged and which is characterized by a potential well of depth $U<0$ and range $l_{\mathrm{v}}$ (in this context, "short-ranged" means that the attractive potential for the monomers decays faster than $1 / z^{1 / v}$ with the distance $z$ of the monomers from the surface; see Refs. [31,32,4]). Adsorption occurs if $|U|$ exceeds a certain threshold value.

It is convenient to introduce the dimensionless variable $u \equiv\left(l_{\mathrm{v}} / a_{\mathrm{po}}\right)^{2} U / T$, which measures the overall strength of the attractive potential, and to first focus on a flat, i.e. planar, membrane surface. The corresponding threshold value for adsorption is denoted by $u=u_{*}<0$. The transition occurs in the limit of large $N$ as the parameter $u$ approaches $u_{*}$ from below. The relevant control parameter is now given by $\epsilon \equiv\left(u-u_{*}\right) /\left|u_{*}\right|$. For $\epsilon>0$ and $\epsilon<0$, the chain is in the desorbed and adsorbed state, respectively.

It has previously been shown for planar surfaces that the two scaling variables at the adsorption transition are given by $N$ and $\epsilon$. The pancake thickness $L_{\perp}$, for example, scales as $L_{\perp}=$ $a_{\mathrm{po}} N^{v} \Omega_{\perp}\left(\epsilon N^{\phi}\right)$ with the dimensionless scaling function $\Omega_{\perp}$ and the crossover exponent $\phi>0$ [33]. For ideal chains or chains in a $\theta$ solvent, one has $\phi=1 / 2$; for self-avoiding chains, $\phi \simeq 0.6$

Using this scaling behaviour as appropriate for a planar surface, one can conclude from a curva- 
ture expansion for the free energy that the polymerinduced curvature has the general scaling form [7]

$M_{\mathrm{pi}}=-\left(T / \kappa a_{\mathrm{po}} N^{v}\right) \Omega_{M}\left(\epsilon N^{\phi}\right)$

where $\Omega_{M}(y)$ again represents a dimensionless scaling function.

For the desorbed state with $y \sim \epsilon>0$, one must recover the behavior as described by Eq. (2) which implies that $\Omega_{M}(y)$ approaches a negative constant, $\Omega_{M}(\infty)<0$, for large positive $y \sim \epsilon$. For the adsorbed state with $\epsilon<0$, on the other hand, the curvature should become independent of $N$ in the limit of large $N$. It then follows from Eq. (4) that $\Omega_{M}(y) \sim y^{v / \phi}$ for large negative $y$, and that $M_{\mathrm{pi}} \sim-T / \kappa a_{\mathrm{po}} \epsilon^{\nu / \phi}$ for $\epsilon<0$ and large $N$. The latter relation is equivalent to Eq. (3) since the pancake thickness $L_{\perp}$ diverges as $L_{\perp} \sim a_{\mathrm{po}} / \epsilon^{v / \phi}$ at the adsorption transition.

Since the mushroom-induced and the pancakeinduced curvatures have opposite signs, the function $\Omega_{M}(y)$ must vanish at a certain value $y=y_{0}$ For ideal chains, we have obtained an explicit expression for the function $\Omega_{M}(y)$ which shows that $y_{0}=0$ for contact potentials, i.e. the curvature switch occurs directly at the adsorption transition [7]. For self-avoiding chains, $y_{0}=0$ also seems to be a rather natural choice, but this remains to be shown. However, even for $y_{0} \neq 0$, the curvature switch at $\epsilon=\epsilon_{0} \equiv y_{0} / N^{\phi}$ will be close to the adsorption transition for long chains since $\epsilon_{0}$ goes to zero for large $N$.

For ideal polymers, it is also possible to obtain explicit expressions for the polymer-induced contributions to the bending rigidity $\kappa$ and to the Gaussian bending modulus $\kappa_{\mathrm{G}}$ for all values of $\epsilon$. For contact potentials, one finds that these excess contributions also change sign at $\epsilon=0$, i.e. directly at the adsorption transition [34].

\section{Polymer-induced adhesion of membranes}

\subsection{Anchored stickers}

In this section, the anchored polymers represent stickers which have a sequence of the form AP...PS. As before, A, P, and S are an anchor, a polar (i.e., water soluble), and a sticky segment of the poly- mer, respectively. These stickers can form bridges between two adjacent membranes which may consist (i) of a single sticker when the sticky segment $\mathrm{S}$ adheres directly to the other membrane, (ii) of two adhering stickers which are bound by their two sticky segments $\mathrm{S}$ and $\mathrm{S}$, or (iii) of two stickers which are connected by an additional linker molecule.

Cases (i) and (ii) are shown schematically in Fig. 5. One example for case (iii) which has recently been studied in some detail is biotin and avidin (or streptavidin) [35,36]. Biotin molecules can be attached to bilayers via lipid anchors. They then form bridges which consist of two anchored

(a)

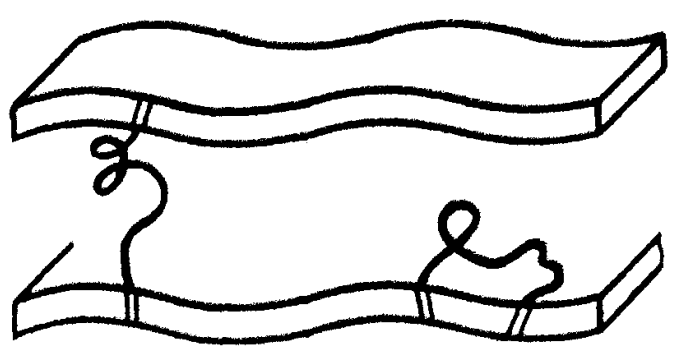

(b)

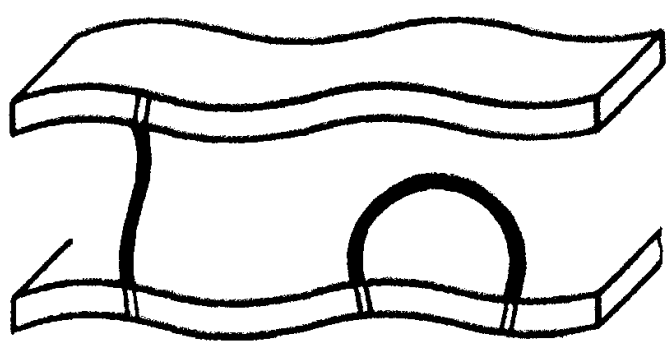

(c)

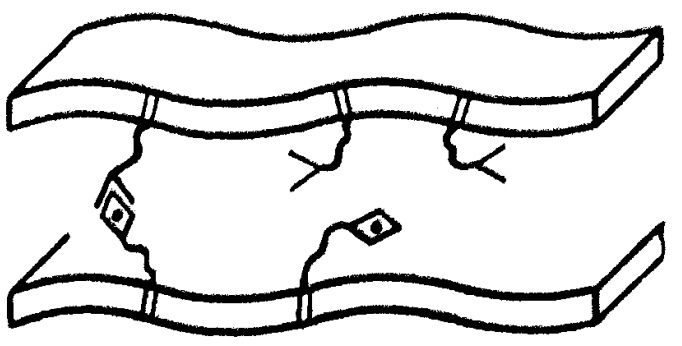

Fig. 5. Pairs of membranes with stickers which can form bridges (left) or arches (right). (a,b) The stickers consist of flexible and rigid polymers, respectively. (c) The bridges are formed from two different types of stickers. 
biotins linked together by an avidin molecule. The corresponding bond energy has been estimated to be $\simeq 35 T$ which is larger than the anchoring energy of the lipid anchor.

Bridges formed by stickers represent the main mechanism which underlies the adhesion of cell membranes. The stickers which are anchored in the cell membranes are cell adhesion molecules (CAMs) or receptor/ligand pairs [37,10]. In the absence of such molecules, cell membranes effectively repel one another [16,38].

Bridges arise from trans-interactions of the stickers in which both ends of one sticker are connected to two different membranes. Stickers may also experience cis-interactions in which both ends of one sticker are linked to the same membrane. The simplest type of cis-interaction is provided by a single sticker which forms an arch (Fig. 5).

\subsection{Arches versus bridges}

Arches are most easily formed if the stickers consist of very flexible polymers, as shown in Fig. 5(a). For a given separation of the membranes, the configurational entropy of such an arch state is larger than the entropy of a bridge state. Therefore, most stickers will form arches and only a small fraction of these stickers will actually form bridges. Since the entropy of the polymer in the arch state attains its maximum in the limit of large separations, these arches lead to a repulsive contribution to the polymer-induced interaction between the membranes which can dominate the attractive contribution arising from the bridges.

In order to suppress the formation of arches, one may use stickers which consist of rigid polymers (Fig. 5(b)): if the bending rigidity of the polymers is sufficiently large, arches become unlikely since the increase in their bending energy dominates over the gain in their entropy. Arches can be avoided completely if the bridges consist of two different stickers forming a receptor/ligand bond and if each type of sticker is only present in one membrane (Fig. 5(c)).

\subsection{Generic and specific interactions between membranes}

The model systems considered in the following consist of lipid bilayers interacting with another membrane or with a rigid wall. The two surfaces cannot penetrate each other and repel one another by generic forces arising, for example, from electrostatic or van der Waals interactions. In addition, these membranes experience specific attractive forces which are mediated by anchored stickers. These stickers are taken to be semi-flexible rodlike molecules as in Fig. 5(b) which do not easily form arches.

The simplest adhesion geometry is provided by one membrane, say 1 , with anchored stickers which interact with another membrane or wall, say 2 , without stickers. The lateral size of one rod-like sticker is denoted by $a_{\|}$, the mean separation of these anchors by $\xi_{\text {an }}$ as before. The sticker concentration (or coverage) is then measured by the area fraction $\bar{\Gamma}_{1} \equiv\left(a_{\|} / \xi_{\text {an }}\right)^{2}$. The adhesion process is controlled, to a large extent, by the magnitude of $\bar{\Gamma}_{1}$

\subsection{Cis-interactions between mobile stickers}

Two stickers, which can move within the same fluid membrane, can approach each other and will then exhibit additional cis-interactions which can be repulsive or attractive. Their overall cis-interaction is effectively repulsive (i) if the intermolecular forces between two stickers are repulsive arising, for example, from electric charges or (ii) if the attractive components of these forces are overcome by the entropy of mixing. In such a situation, each membrane, consisting of a mixture of lipids and anchored stickers, is in a homogeneous state, i.e. in a state with uniform composition.

On the other hand, if the cis-interactions between two stickers are effectively attractive, these stickers may form clusters or domains which leads to new adhesion phenomena (in general, the phase boundaries for the intramembrane states will also be affected by the trans-interactions of the stickers). These two different cases will be discussed in the next two subsections.

\subsection{Adhesion thresholdfor sticker concentration}

The stickers are now taken to repel one another within the same membrane which implies that they do not form clusters or domains. Each sticker can form a bridge, i.e. a localized and short-ranged 
adhesive bond. This intermembrane attraction arising from the stickers is reduced by the shape fluctuations or undulations of the membranes. As shown in Ref. [8], this reduction leads to an adhesion threshold: the effective strength $|U|$ of the sticker-mediated attraction must exceed a certain threshold value in order to overcome the fluctuation-induced repulsion.

The effective strength $|U|$ depends on the sticker concentration $\bar{\Gamma}_{1}$; for small $\bar{\Gamma}_{1}$, one has $|U| \sim \bar{\Gamma}_{1}$ Therefore, the sticker concentration must also exceed a certain threshold $\bar{\Gamma}_{\mathrm{c}}$ before adhesion sets in. Furthermore, if the concentration $\bar{\Gamma}_{1}$ approaches the critical value $\bar{\Gamma}_{\mathrm{c}}$ from above, the membranes undergo a continuous unbinding transition and their mean separation diverges as a power law, as follows from previous work on the unbinding transitions of membranes $[39,40]$.

As shown in Ref. [8], the critical concentration $\bar{\Gamma}_{\mathrm{c}}$ is maximal for tensionless membranes and is then given by

$$
\bar{\Gamma}_{\mathrm{c}}(0) \simeq(T / \kappa)\left(a_{\|} / l_{1}\right)^{2} /\left[\exp \left(a_{\|}^{2}\left|U_{1}\right| / T\right)-1\right]
$$

where $l_{1}$ and $\left|U_{1}\right|$ are the effective potential range and the effective potential strength, respectively, arising from a single sticker molecule. For phospholipid bilayers with $\kappa \simeq 10^{-19} \mathrm{~J}$, this expression leads to the estimate $\bar{\Gamma}_{\mathrm{c}}(0) \lesssim 10^{-2}$ at room temperature.

In the presence of a lateral tension $\Sigma$, the adhesion threshold decreases since the tension acts to reduce the shape fluctuations of the membranes. More precisely, the critical concentration is found to decrease as

$\bar{\Gamma}_{\mathrm{c}}(\Sigma) \approx \bar{\Gamma}_{\mathrm{c}}(0)\left(1-c \sqrt{\Sigma / \Sigma_{*}}\right)$

where $c$ is a dimensionless coefficient of order one. This relation should hold up to the characteristic tension $\Sigma=\Sigma_{*} \simeq T / l_{1}^{2}$. For $\Sigma \gtrsim \Sigma_{*}$, the threshold concentration $\bar{\Gamma}_{\mathrm{c}}(\Sigma)$ decays rapidly to zero. For a potential range $l_{1} \simeq 3 \mathrm{~nm}$, one has $\Sigma_{*} \simeq 0.02 \mathrm{~mJ} \mathrm{~m}^{-2}$ at room temperature; for many bilayer systems, the lateral tension is estimated to be below this value which implies that these systems should indeed exhibit a finite adhesion threshold $\bar{\Gamma}_{\mathrm{c}}$.

\subsection{Adhesion via sticker-rich domains}

If the cis-interactions between two mobile stickers within the same membrane are effectively attractive, the stickers may form clusters or domains which leads to new adhesion phenomena. Thus, let us assume that the mixed bilayer has been prepared in a two-phase region in which a sticker-poor phase, say $\alpha$, coexists with a stickerrich phase, say $\beta$. If such a membrane undergoes phase separation, one has $\alpha$ domains with a small sticker concentration $\bar{\Gamma}_{1}=\bar{\Gamma}_{\alpha}$ and $\beta$ domains with a larger sticker concentration $\bar{\Gamma}_{1}=\bar{\Gamma}_{\beta}$. When this membrane interacts with another membrane without stickers, the effective sticker attraction $|U|$ is now given by $\left|U_{\alpha}\right| \sim \bar{\Gamma}_{\alpha}$ and $\left|U_{\beta}\right| \sim \bar{\Gamma}_{\beta}$, respectively.

In general, both interaction strengths will differ from each other and may lie above or below the adhesion threshold $\left|U_{\mathrm{c}}\right|$. This leads to different mean separations for the different membrane domains. One interesting case is obtained if $\left|U_{\alpha}\right|<\left|U_{\mathrm{c}}\right|$, but $\left|U_{\beta}\right|>\left|U_{\mathrm{c}}\right|$. In the latter situation, the interacting membranes only adhere via the $\beta$ domains, as shown schematically in Fig. 6. This resembles the focal adhesions which are often observed for cell membranes.

\subsection{Extensions}

It is straightforward to generalize the above theory to two fluid membranes which both contain stickers. If the bonds or bridges between the membranes are formed by two adhering stickers, the effectivestrength $|U|$ of the sticker-mediated attraction is proportional to $\bar{\Gamma}_{1} \bar{\Gamma}_{2}$ where $\bar{\Gamma}_{1}$ and $\bar{\Gamma}_{2}$ are the sticker concentrations in membrane 1 and 2 , respectively. Likewise, if these bridges consist of two stickers connected by one linker molecule,

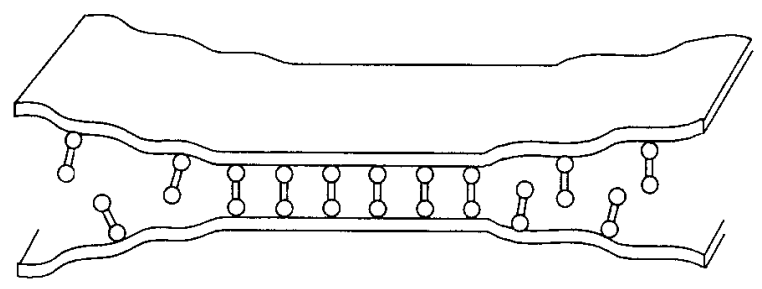

Fig. 6. Phase separation of the membrane into sticker-poor domains with sticker concentration $\Gamma=\Gamma_{\alpha}<\Gamma_{\mathrm{c}}$ and sticker-rich domains with $\Gamma=\Gamma_{\beta}>\Gamma_{\mathrm{c}}$ 
the effective strength $|U|$ is proportional to $\bar{\Gamma}_{1} \bar{\Gamma}_{2} p(1-p)$ where $p$ is the fraction of stickers with one bound linker molecule. Phase separation within the interacting membranes now leads to three possible values $\left|U_{\alpha \alpha}\right|\left|U_{\alpha \beta}\right|$ and $\left|U_{\beta \beta}\right|$ for the sticker-mediated attraction, and, thus, to three values for the mean separations of the different pairs of interacting membrane domains.

Other systems of interest are provided by membranes with anchored stickers which are immobilized. Two cases can be distinguished. First of all, the stickers may be immobilized via their sticky segments. Such a segment may be strongly bound, for example to a solid surface or to a polymer network (such as the cytoskeleton). In such a situation, the sticker positions are quenched or frozen even though the membrane may still be in a fluid state. On the other hand, the stickers are also immobilized if their anchors can no longer diffuse along the membrane. This happens, for example, if the stickers are anchored in a polymerized or solid-like membrane. The quenched disorder in the sticker positions arising from this immobilization leads to quenched disorder in the attractive intermembrane potential. The latter type of disorder represents a marginal and irrelevant perturbation at the unbinding transition of fluid and polymerized membranes, respectively [41]. The adhesion threshold and the critical behavior for these systems remain to be studied $^{1}$.

\section{Acknowledgment}

I thank Christin Hiergeist and Vasudevamurthy Indrani for enjoyable collaborations.

\section{References}

[1] Structure and dynamics of membranes, in: R. Lipowsky, E. Sackmann (Eds.), Handbook of Biological Physics, Vol. 1, Elsevier, Amsterdam, 1995.

[2] P.-G. de Gennes, Scaling Concepts in Polymer Physics, Cornell University Press, Ithaca, 1979.

\footnotetext{
${ }^{1}$ The behaviour discussed in Sections 3.4-3.7 also applies to other types of stickers such as oppositely charged lipids. In addition, wetting layers with stickers exhibit the same critical behaviour as tense membranes with stickers.
}

[3] M. Doi, S.F. Edwards, The Theory of Polymer Dynamics, Clarendon Press, Oxford, 1986.

[4] E. Eisenriegler, Polymers Near Surfaces, World Scientific, Singapore, 1993.

[5] R. Lipowsky, Europhys. Lett. 30 (1995) 197.

[6] C. Hiergeist, R. Lipowsky, J. Phys. France 6 (1996) 1.

[7] C. Hiergeist, V. Indrani, R. Lipowsky, Europhys. Lett. 36 (1996) 491

[8] R. Lipowsky, Phys. Rev. Lett. 77 (1996) 1652

[9] J. Darnell, H. Lodish, D. Baltimore, Molecular Cell Biology, Scientific American Books, Freeman and Company, New York, 1990.

[10] B. Alberts et al., Molecular Biology of the Cell, 3rd ed., Garland, New York, 1994.

[11] G. Blume, G. Cevc, Biochim. Biophys. Acta 1029 (1990) 91.

[12] D. Lasic et al., Biochim. Biophys. Acta 1070 (1991) 187.

[13] A. Kenworthy, S. Simon, T. McIntosh, Biophys. J. 68 (1995) 1903.

[14] H. Ringsdorf, J. Simon, F.M. Winnik, in: P.L. Dubin, P. Tong (Eds.), Colloid-Polymer Interactions, Vol. 532 of ACS Symp. Ser., American Chemical Society, Washington, DC, 1993, pp. 216-240.

[15] J. Simon, M. Kühner, H. Ringsdorf, E. Sackmann, Chem. Phys. Lipids 76 (1995) 241.

[16] G.I. Bell, Science 200 (1978) 618.

[17] F. Brochard, J. Lennon, J. Phys. (Paris) 36 (1975) 1035.

[18] E. Evans, A. Yeung, R. Waugh, J. Song, in: R. Lipowsky, D. Richter, K. Kremer (Eds.), The Structure and Conformation of Amphiphilic Membranes, Vol. 66 of Springer Proceedings in Physics, Springer, Berlin, 1992, pp. 148-153.

[19] U. Seifert, S. Langer, Biophys. Chem. 49 (1994) 13.

[20] W. Bancroft, C. Tucker, J. Phys. Chem. 31 (1927) 1681.

[21] W. Helfrich, Z. Naturforsch. 28c (1973) 693.

[22] H. Hadwiger, Vorlesungen über Inhalt, Oberfläche und Isoperimetrie, Springer, Berlin, 1957.

[23] C. Likos, K. Mecke, H. Wagner, J. Chem. Phys. 102 (1995) 9350

[24] S. Alexander, J. Phys. (Paris) 38 (1977) 983.

[25] P.-G. de Gennes, Macromolecules 13 (1980) 1069.

[26] M. Daoud, J. Cotton, J. Phys. (Paris) 43 (1982) 531.

[27] S. Milner, T. Witten, J. Phys. France 49 (1988) 1951.

[28] K. Hristova, D. Needham, J. Colloid Interface Sci. 168 (1994) 302.

[29] P.-G. de Gennes, J. Phys. Chem. 94 (1990) 8407.

[30] J. Brooks, C. Marques, M. Cates, J. Phys. II (Paris) 1 (1991) 673.

[31] R. Lipowsky, A. Baumgärtner, Phys. Rev. A 40 (1989) 2078.

[32] R. Lipowsky, Phys. Scripta T29 (1989) 259.

[33] E. Eisenriegler, K. Kremer, K. Binder, J. Chem. Phys. 77 (1982) 6296.

[34] V. Indrani, R. Lipowsky, in preparation.

[35] E.L. Florin, V.T. Moy, H.E. Gaub, Science 264 (1994) 415.

[36] S. Chiruvolu et al., Science 264 (1994) 1753.

[37] T.A. Springer, Nature 346 (1990) 425.

[38] G.I. Bell, M. Dembo, P. Bongrand, Biophys. J. 45 (1984) 1051.

[39] R. Lipowsky, S. Leibler, Phys. Rev. Lett. 59 (1987) 1983.

[40] R. Lipowsky, Europhys. Lett. 7 (1988) 255.

[41] R. Lipowsky, U. Seifert, Langmuir 7 (1991) 1867. 